\title{
CONVERGÊNCIA DAS REDES DE COMUNICAÇÃO: ASPECTOS TÉCNICOS E ECONÔMICOS
}

\author{
Antônio Teófilo Nassif ${ }^{1}$ Antonio José Martins Soares ${ }^{2}$ \\ Recibido el 24 de septiembre de 2004, aceptado el 9 enero de 2005
}

\begin{abstract}
RESUMO
A tendência nos sistemas de comunicações mundiais é a convergência das redes tradicionais de telefonia, wireless e Internet, hoje providas separadamente, para uma única infra-estrutura de transmissão em pacotes. Da convergência para uma infra-estrutura inteligente e altamente eficiente, derivarão acesso universal e uma gama de novas oportunidades para tecnologias, aplicações e serviços. As informações apresentadas neste trabalho demonstram que, em futuro próximo, as operadoras tradicionais deverão agregar valor aos seus serviços para manterem o mesmo nível de receita atual ou aumentarem a sua rentabilidade. Para isso, deverão implantar uma rede convergente, seja gradativamente, aproveitando os equipamentos existentes, ou radicalmente, substituindo totalmente a sua planta.
\end{abstract}

Palavras chave: Redes de comunicação, convergência de redes, arquitetura de rede convergente.

\section{ABSTRACT}

The tendency in world communications is the convergence of the three traditional networks (voice network, wireless network and data network), currently provided separately, into a common packet based infrastructure. Universal access and a host of new technologies, applications and services will be derived from the convergence into an intelligent and highly efficient infrastructure. This work presents information demonstrating that, in the near future, established operators, in order to keep up current revenues and to increase profitability, should add value to services. For this purpose, they should deploy the convergence in a gradual manner, taking advantage of existing assets, as well as from the scratch, fully replacing their entire TDM infrastructure.

Keywords: Communications networks, convergence network.

\section{INTRODUÇÃO}

A indústria de telecomunicações procura, há alguns anos, orientar sua tecnologia de maneira a tornar os operadores competitivos em um ambiente caracterizado pela concorrência e aumento da desregulamentação. As redes de comunicação convergentes, com interfaces abertas e capacidade para transmitir voz, dados, imagens, som e vídeo, exploram plenamente as tecnologias de ponta para oferecer serviços sofisticados e aumentar as receitas dos operadores, reduzindo despesas de investimentos e custos de operação [1].

A principal diferença entre as redes convergentes e as redes tradicionais de comutação por circuitos está na estrutura de transmissão por pacotes utilizada no protocolo Internet (IP) e adotada nessas novas redes. Os terminais encaminham pacotes de dados, em formato IP, para um ponto concentrador, de onde passam a circular pela rede até encontrar o endereço IP de destino. Basicamente, é o mesmo procedimento hoje em uso na Internet. A principal mudança a ser feita na estrutura das operadoras para oferecer serviços convergentes, referese à transmissão de voz. Para que possam trafegar nas novas redes, os sinais de voz precisam ser transformados em pacotes, que se misturam aos pacotes de dados e imagens durante o transporte. Essa função é realizada por gateways de voz, que são instalados na camada de transporte da rede, onde também estão os roteadores e toda a infra-estrutura física da operadora. $\mathrm{O}$ uso de interfaces e protocolos abertos e padronizados é uma das grandes vantagens das redes convergentes. Além disso, a sua arquitetura dispensa algumas estruturas convencionais, como as centrais de trânsito.

De modo geral, os provedores dividem a arquitetura das redes convergentes em pelo menos três camadas básicas: infra-estrutura de transporte e acesso, controle de

\footnotetext{
1 Empresa Brasileira de Correios e Telégrafos (ECT), Brasília - DF - Brasil, ANTONIO@correios.com.br

2 Departamento de Engenharia Elétrica, Universidade de Brasília (UnB), Brasília - DF - Brasil, martins@ene.unb.br
} 
chamadas e serviços. Na primeira, estão as unidades de acesso de assinantes, como telefones IP e access gateways (uma espécie de armário multiprotocolo que faz a interface entre a rede IP e os diferentes tipos de conexão do usuário, como circuito de voz, linha ADSL etc.), além de comutadores, roteadores e media gateways, que transformam sinais de voz da rede convencional em pacotes. A camada de controle de chamadas é a responsável pelo encaminhamento, supervisão e liberação das ligações que trafegam pela rede IP (ou ATM). É uma parte estratégica, onde fica o elemento responsável pela inteligência das redes: o softswitch, ou media gateway controller. Considerada o grande diferencial e o atrativo das redes convergentes, a camada de serviços é formada pelos softwares que vão permitir às operadoras oferecer novos e múltiplos serviços aos usuários.

As redes convergentes têm introduzido uma nova organização no tocante, principalmente, aos planos de transporte e de controle. Para explorar esse conceito, é preciso desenvolver diferentes aplicações ou enriquecer as existentes. Elas vão trazer a coerência necessária entre as aplicações tradicionais, do mundo da telefonia, e as do mundo de dados, graças à utilização de um plano de transporte fundamentado sobre o IP e à separação das camadas de transporte, controle e aplicação. De outra forma, a cooperação entre estes dois mundos vai sem dúvida harmonizar as funcionalidades de ligação de um ao outro mundo, como as mensagens textuais, as mensagens vocais, a localização geográfica ou a presença. Enfim, essa harmonização permite às aplicações existentes ou às novas aplicações tirar partido das funcionalidades desses dois mundos.

A evolução de uma rede existente em direção a essa nova estrutura necessitará de uma estratégia de migração progressiva, visando uma redução ao mínimo das despesas de investimentos durante a fase de transição e se beneficiando das vantagens que ela apresenta. Toda iniciativa empreendida, quando dessa etapa de transição, deverá simplificar a evolução da rede em direção à arquitetura convergente e à comutação de pacotes. Durante vários anos ainda, os serviços de comutação tradicional vão coexistir com os elementos de rede da nova tecnologia.

\section{CONVERGÊNCIA DE VOZ E DADOS}

A convergência apresenta uma nova visão sobre o futuro das redes de comunicação e de aplicações multimídia. Será concretizado o velho sonho de uma plataforma de transporte comum para vídeo, voz e dados. A convergência de redes permitirá aplicações do tipo telefonia via IP, acesso a Web através de telefones móveis e o streaming de vídeo se tornará uma realidade. Os protocolos da Internet suportam o transporte de dados de praticamente qualquer tipo de rede, desde redes locais (LAN) até redes globais com vários provedores de redes, embora as tradicionais redes de dados, como o SNA da IBM e as redes legadas de alguns provedores de telecomunicações (X.25, frame relay), estejam fazendo um grande esforço para se acomodarem nas redes IP.

Um dos desafios da convergência é a complexidade de testes para a determinação de problemas e garantia de disponibilidade e performance [2]. Isso é importante para reduzir os custos de operação e o número de reparos e melhorar o tempo de re-estabelecimento dos serviços, possibilitando antecipar e evitar a ruptura do serviço por meio de um eficiente processo de coleta de eventos e uma constante monitoração da rede.

A integração de recursos e a convergência do tráfego reduzem os custos totais da rede, permitindo o compartilhamento da operação, a administração, a manutenção de equipamentos e facilidades para o desenvolvimento de aplicações multimídia. As tecnologias da Internet oferecem oportunidades para combinar os serviços de voz, dados e vídeo, criando sinergia entre eles.

Na Fig. 1 mostrase os elementos de uma rede projetada para suportar comunicação de voz e transporte de dados. De fato, as características de implementação dessa rede podem variar como resultado de diferentes tecnologias, padrões conflitantes e definições de serviços com diferentes QoS (Quality of Service). Redes de melhor esforço são inadequadas para os serviços da próxima geração, como multimídia e comércio eletrônico, que demandam uma alta QoS. Contudo, tais redes são suficientes para outros serviços como e-mail e aqueles embasados em protocolo de transferência de arquivos como FTP.

Cada serviço pode ser diferenciado por um acordo de nível de serviço (SLA) que define parâmetros como QoS e largura de banda. Os SLAs por si só não surtem qualquer efeito, pois seu valor reside na maneira que são gerenciados na rede. É essencial melhorar a habilidade do provedor de serviço para fazer cumprir o contrato com o cliente, como definido no SLA, de sorte a se fazer uso otimizado da rede, minimizando a aplicação de penalidades por não aderência. 
A diferenciação do provedor de serviço dependerá fortemente de seu gerenciamento e monitoração do SLA e a crescente confiança depositada pelo cliente. Conseqüentemente, o gerenciamento do SLA é um diferencial na oferta de serviços pelo provedor.

Como o modelo de negócios está evoluindo do provimento de infra-estrutura de rede para o fornecimento de serviços, necessita-se definir QoS e suporte ao gerenciamento do SLA, tirando proveito das informações intrínsecas à rede. A introdução de redes convergentes deve ser postergada se nenhum suporte ao gerenciamento for provido, deixando os clientes inseguros quanto à confiabilidade do serviço ofertado.

Os principais desafios do gerenciamento são: prover um serviço confiável e aderente ao SLA; monitorar completamente o SLA enquanto o serviço está sendo provido; reagir rapidamente caso o desempenho esteja insatisfatório; antecipar, tanto quanto possível, qualquer queda no desempenho, focando na previsão de desempenho do serviço; e assegurar faturamento acurado com base no conteúdo e QoS [3].
A rede convergente pode ser considerada como uma associação de redes autônomas operadas por autoridades diferentes, cada qual com sua própria forma de disponibilizar o serviço (fixo, acesso móvel, óptica etc.). Os componentes da rede que contribuem para disponibilizar um serviço não são tratados necessariamente pelo mesmo provedor de serviço. De uma abordagem fim-a-fim, isso implica que SLAs têm que ser estabelecidos entre provedores, assim como entre clientes e provedores. Então, SLAs devem ser gerenciados em níveis diferentes, desde a camada 7 (provedor de serviços de aplicação) até a camada 2 (transporte óptico) e em diferentes domínios (acesso/usuários finais, núcleo/acesso, núcleo/núcleo etc.).

Um desafio maior é o gerenciamento de SLA em domínios cruzados, o que é executado com a propagação de SLA de uma extremidade à outra. Para reduzir o tempo de desenvolvimento, é necessário definir um SLA padrão e protocolo de negociação entre provedores que simplificarão a propagação de SLA na rede.

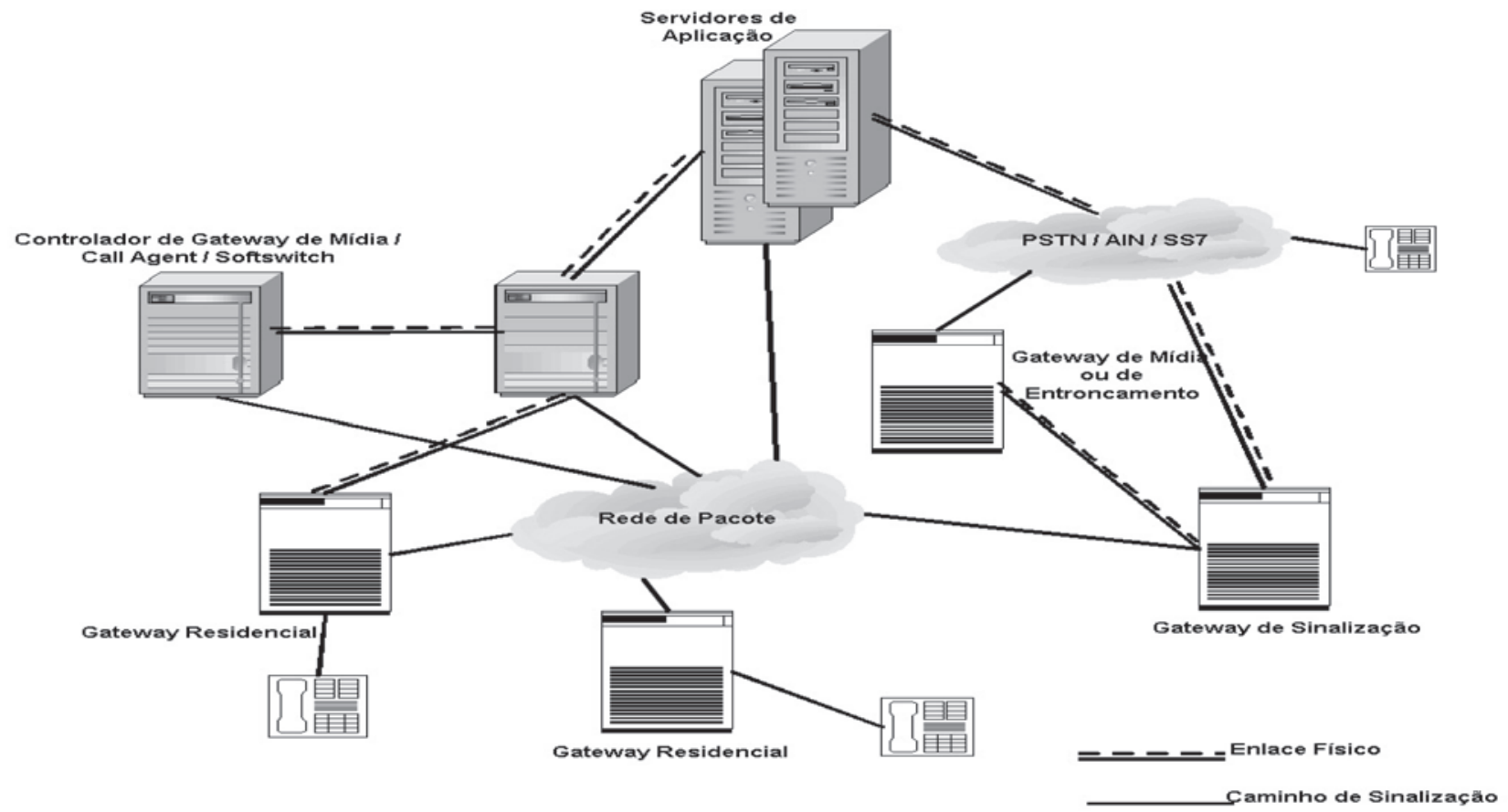

Fig.1 Rede de voz e dados convergente. 


\section{ARQUITETURA DA REDE CONVERGENTE}

A arquitetura da rede convergente representada na Fig. 2 repousa sobre o transporte de voz e de dados em forma de pacotes. Ela decompõe os blocos monolíticos dos comutadores atuais em diferentes camadas de rede que interfuncionam por intermédio de interfaces abertas. A inteligência de tratamento de chamada via comutador da rede telefônica pública comutada (RTPC) é dissociada do hardware da matriz de comutação. Esta inteligência agora reside em um equipamento distinto: o softswitch ou servidor de chamada (media gateway controller ou agente de chamada), que dentro da nova arquitetura é o elemento de controle. As interfaces abertas em direção às aplicações de rede inteligentes e dos novos servidores de aplicação facilitam o fornecimento rápido de serviços e a diminuição dos atrasos de comercialização.

Na camada media, são introduzidos media gateways para adaptar a telefonia e outras mídias à rede de transporte de pacotes. Os media gateways são usados para interfacear seja com os equipamentos dos usuários (gateway residencial) com acesso à rede (gateway de acesso), ou com a RTPC (gateway tronco). Servidores de media especiais implementam uma variedade de funções, como fornecimento de tom de discagem ou de anúncio. Funções mais avançadas de servidores de media incluem resposta interativa de voz e conversão de voz para texto e texto para voz.
A interface aberta para essa nova arquitetura permite introduzir rapidamente novos serviços. Ao mesmo tempo, elas facilitam a introdução de modelos de novos negócios quebrando a cadeia de valores tradicionais de hoje em vários serviços os quais podem ser fornecidos por diferentes provedores.

Na Fig. 3 mostrase os componentes e sua relação um com o outro para formar a arquitetura da rede convergente [4]. Tecnologias como DWDM (Dense Wavelength Division Multiplexing) e MPLS (MultiProtocol Label Switching) formarão a infra-estrutura básica que interconectará todos os nós. MPLS será usado para segregar os diferentes tipos de dados que serão transportados pela infra-estrutura. Os dados serão de dois tipos: media streams (voz, vídeo e outras formas de conteúdo) e dados de controle (sinalização).

Acima da camada de transporte estará a camada de transporte de sinalização, carregando todas as mensagens de controle para/e do gateway media e os seus gateway controller media, encapsulado a sinalização número 7 do sistema de sinalização (SS7), mensagens carregadas dos gateways de sinalização para os media gateway controllers e mensagens do protocolo de iniciação de sessão (SIP) entre clientes SIP e proxy e servidores de aplicação SIP.

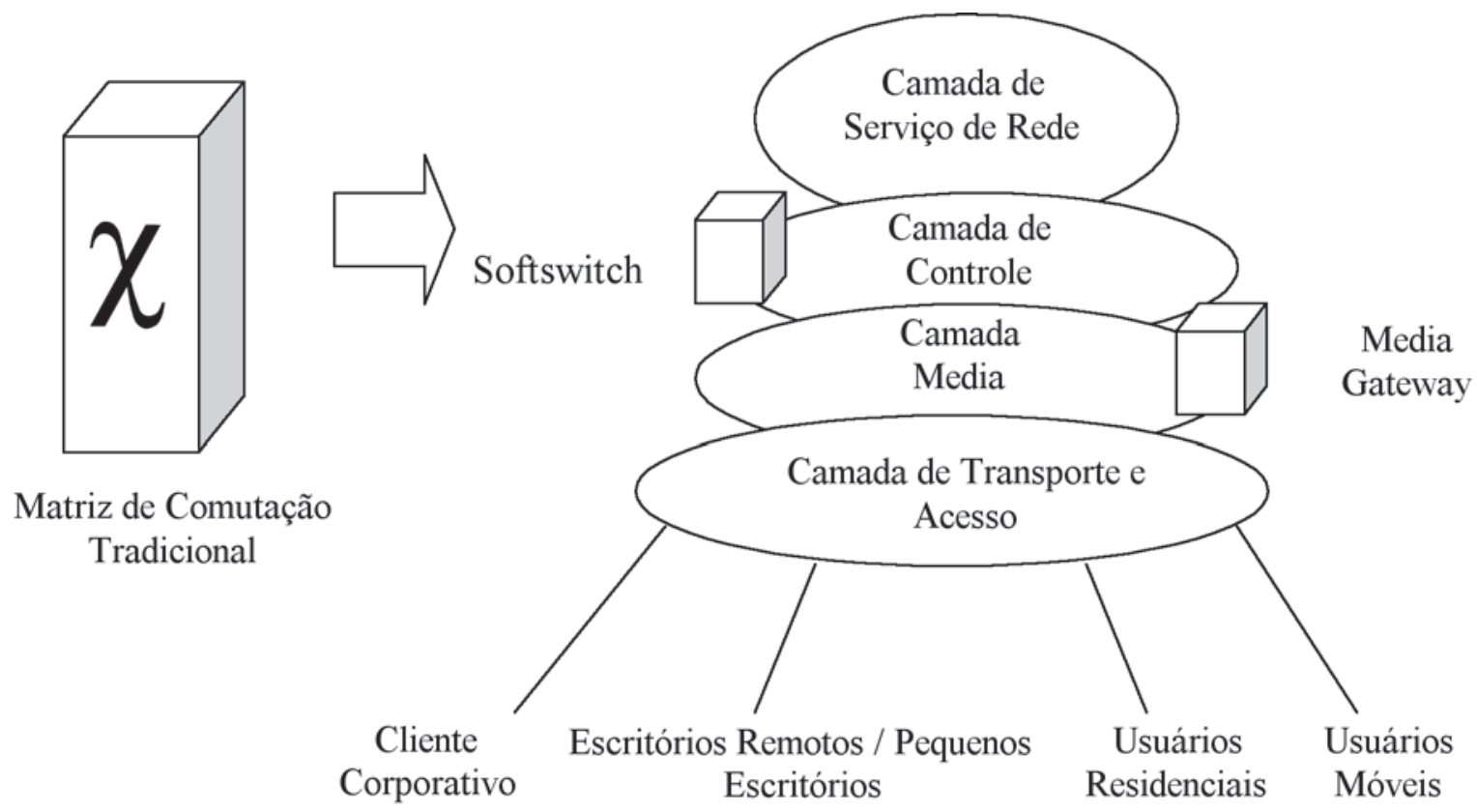

Fig. 2 Arquitetura da rede convergente em camadas. 
A camada superior (mais alta) é onde todos os servidores residem para permitir uma comunicação rápida entre aplicações distribuídas e entre diferentes plataformas de serviços. Esta camada carregará todas as informações para os sistemas de suporte operacional e de negócios para manutenção e taxação, e conterá todos os diretórios e base de dados que armazenará as informações de locação e configuração de assinantes/usuários e os serviços. Esta camada também poderia fixar o ponto de controle do serviço de uma rede inteligente convencional comutada por circuito. Nesse caso, o ponto de controle teria implementado uma interface do IP e estaria comunicando (protocolo de controle da transmissão via stream) com o media gateway controller ou softswitch mascarado como um ponto de comutação do serviço. Isto proveria uma integração do serviço da rede antiga e da nova rede convergente.

As partes finais e mais importantes da rede serão os firewalls e os roteadores, que interconectarão as redes de diferentes operadoras e provedoras de serviços. A Fig. 3 mostra como os firewalls e os roteadores de filtro de pacotes estão conectados.

\section{MIGRAÇÃO PARA A REDE CONVERGENTE}

A Internet atual se esforça a dar vazão à totalidade de tráfego o mais rapidamente possível dentro do limite de sua capacidade, mas sem nenhuma garantia quanto à vazão, ao atraso de transmissão, às variações deste atraso (jitter) e à taxa de perda de pacotes. Até o presente, o paradigma do encaminhamento de melhor esforço tem funcionado bem porque a maior parte das aplicações de dados IP são pouco prioritárias, ocupam pouca largura de banda e são tolerantes a atraso de transmissão e ao jitter. Isso está mudando rapidamente. As aplicações Internet multimídias interativas em tempo real (telefonia e vídeo) e as redes privadas virtuais requerem uma largura de banda garantida e/ou atrasos restritos. Ainda mais, o número de usuários da Internet aumenta de uma maneira exponencial, o que faz crescer o risco de congestionamento, de retardo e de perda de pacotes. A principal dificuldade é dotar as redes IP existentes de capacidade de multisserviços escaláveis, conservando as vantagens da rede IP, que tornou possível a Internet. Nestas redes multisserviços, as operadoras devem honrar os contratos de nível de serviço complexos, que definem as bandas passantes e outros parâmetros de qualidade para diferentes tipos de tráfego. É a qualidade de serviço IP.
A arquitetura da rede convergente oferece a oportunidade de não somente atender esses requisitos tecnológicos, mas também aumentar o lucro e reduzir os custos de investimentos e operacionais.

As novas operadoras não necessitam elaborar uma estratégia de migração, porque desde o início elas podem optar por uma solução de convergência de voz e dados para propiciar serviços avançados de voz e dados. Em contraste, as fornecedoras de serviços já estabelecidas devem considerar o seu parque de equipamentos TDM e são colocadas em uma situação delicada: fazer evoluir os seus comutadores de circuitos existentes (se isto tem sido previsto pelo fornecedor), parar com os investimentos em equipamentos de comutação de circuitos e construir uma rede convergente superposta, ou ainda substituir gradativamente os comutadores de circuitos existentes. Deve ser igualmente considerado o impacto do tráfego Internet, com as suas longas durações de ocupação, o que congestiona a rede de comutação de circuito, concebida em sua origem para vender tráfego telefônico com tempo de ocupação muito curto.

Para tornarem-se competitivas, as operadoras devem encontrar maneiras de fornecer novos serviços à sua clientela durante a fase de transição até atingir a evolução para a convergência. Se houver erro na estratégia de migração, a operadora terá que assumir as conseqüências em longo prazo de seu mau investimento. Mas, se escolher uma boa estratégia, ela poderá bem antes de seus concorrentes propor a nova geração de serviços a seus clientes. Infelizmente, não há fórmula mágica sobre quando e como operar essa migração. Tudo depende do estado da rede existente, das exigências da clientela e, de modo geral, dos planos de desenvolvimento da operadora da rede.

A implementação de uma infra-estrutura de rede convergente para o provimento de serviços de voz e dados integrados, em contraste com as atuais plataformas independentes, representa um enorme potencial de redução de custos de operação e manutenção de rede. Verifica-se que, na atual topologia de rede, quanto maior a diversidade de serviços associados, maior será a quantidade de elementos e a complexidade da rede. Mas as redes convergentes, ao contrário, possibilitam uma redução de até $80 \%$ dos elementos de rede de comutação, resultando em até $40 \%$ de redução nos custos operacionais e de manutenção da rede [5]. 
Desse modo, as soluções associadas a redes convergentes possibilitam a minimização de investimentos em infraestrutura, a redução de custos de operação e manutenção e a ampliação de fontes de receitas, por meio do provimento de novos serviços como aplicações multimídia, VoIP e portais de voz, além de um novo leque de acordos de níveis de serviço com base na facilidade de alocação dinâmica de banda.

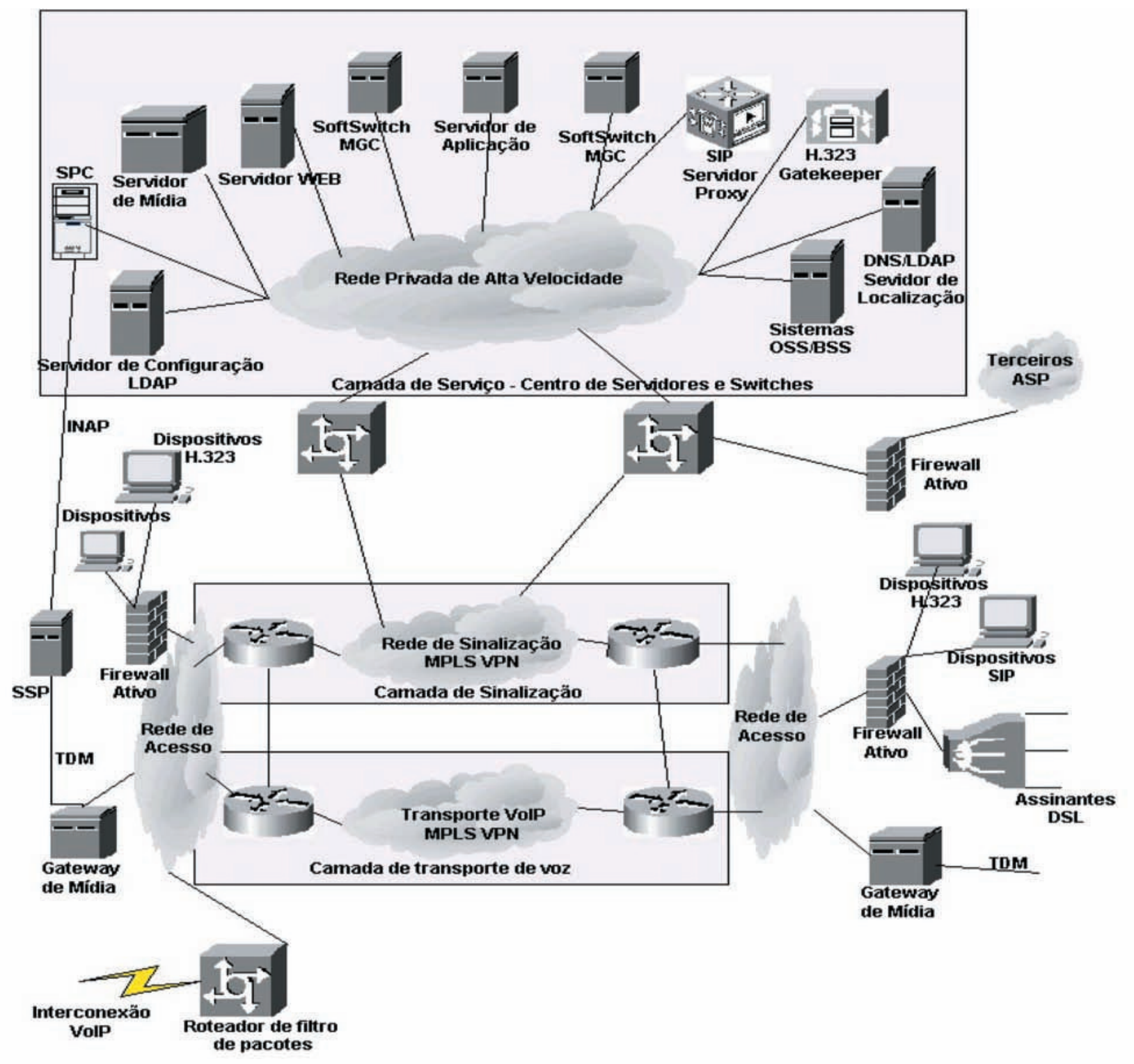

Fig. 3 Arquitetura da rede convergente. 


\section{CONCLUSÕES}

A indústria de telecomunicações está mudando rapidamente. Se os serviços telefônicos constituem a principal fonte de receita dos provedores de serviços estabelecidos, este mercado não se desenvolve tão rapidamente para absorver sem prejuízo os novos operadores. Os provedores de serviços estabelecidos devem então encontrar novas maneiras de conservar uma clientela que os operadores emergentes tentam tirar. Aúnica maneira de manter os clientes e ganhar novos será a de lhes propor serviços correspondentes às suas necessidades, isto é, combinar voz e dados de uma maneira flexível e econômica. Os provedores de serviços e os operadores de rede vão necessitar de sistemas abertos para sobreviver.

Fabricantes de equipamentos e sistemas já oferecem uma estratégia de produtos convergente, que são fundamentados na evolução de produtos existentes e focados nos novos serviços. Essa estratégia, atualizada constantemente para seguir a evolução do mercado, permite fornecer a qualquer operador a solução mais apropriada para realizar a migração suave dos serviços telefônicos para a rede convergente.

As redes convergentes, com o ambiente IP, simplicidade, flexibilidade e abertura, permitirão às operadoras oferecer novos serviços, aplicações e comodidade aos seus usuários de uma maneira mais eficiente e de custo mais otimizado do que se adotasse uma rede de serviços por comutação de circuitos.

Enfim, a rede convergente com QoS definida terá um impacto decisivo nos modelos de negócios das operadoras de serviços de telecomunicações, gerando soluções mais competitivas, com maior eficiência de utilização de rede e com a sua infra-estrutura orientada à demanda de tráfego e a conjuntos completos e amplos de novos serviços de multimídia e de banda larga.
A migração para a arquitetura convergente ocorre, basicamente, em três etapas. A primeira é a adequação das centrais de trânsito, responsáveis pela conexão das centrais de comutação locais ao sistema de voz sobre IP. Isso se faz por meio de gateways, cuja função é empacotar a voz e jogá-la na rede de dados, etapa do processo que algumas operadoras já superaram. A segunda compreende o transporte dos sinais de voz em pacotes até a casa do assinante por meio dos gateways de acesso, substituindo as centrais locais. A terceira, ainda distante, compreende a instalação de telefones IP na mesa de cada usuário.

\section{REFERÊNCIAS}

[1] A.T. Nassif. "Redes da próxima geração: aspectos técnicos, econômicos e cenários de migração". Dissertação de Mestrado. Departamento de Engenharia Elétrica, Universidade de Brasília, Brasília, DF, 2004: 164.

[2] J. Ryan. "Testing and analysing next generation networks". http://www.acme-ids.org, 2002.

[3] G. Désoblin e H. Papini. "SLA management: a key differentiator for service providers". Alcatel Telecommunications Review, $3^{\text {rd }}$ Quarter, 2001.

[4] N. Wilkison. "Next generation network service, technologies and strategies". John Wiley \& Sons Ltd., 2002.

[5] Siemens Comunicação Corporativa. "NGN: em busca do retorno desejado". http://siemens.com.br. Acesso em 15/10/02. 